Research Paper

\title{
Changes in Follicular Helper T Cells in Idiopathic Thrombocytopenic Purpura Patients
}

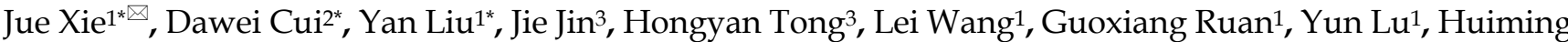 \\ Yuan ${ }^{1}$ \\ 1. Department of Blood Transfusion, the First Affiliated Hospital, School of Medicine, Zhejiang University, Hangzhou, China, 310003 \\ 2. Department of Clinical Laboratory, the First Affiliated Hospital, School of Medicine, Zhejiang University, Hangzhou, China, 310003 \\ 3. Department of Hematology, the First Affiliated Hospital, School of Medicine, Zhejiang University, Hangzhou, China, 310003 \\ ${ }^{*}$ Equal contributors
}

Corresponding author: Jue Xie, Department of Blood Transfusion, the First Affiliated Hospital, School of Medicine, Zhejiang University, Hangzhou, China, Tel:+0571-87236391, Fax: +0571-87236391, Email:zyyyxj2011@163.com

() Ivyspring International Publisher. This is an open-access article distributed under the terms of the Creative Commons License (http://creativecommons.org/ licenses/by-nc-nd/3.0/). Reproduction is permitted for personal, noncommercial use, provided that the article is in whole, unmodified, and properly cited.

Received: 2014.07.23; Accepted: 2014.11.30; Published: 2015.01.05

\begin{abstract}
Background: Idiopathic thrombocytopenic purpura (ITP) is a primary autoimmune disease with a decreased platelet count caused by platelet destruction mediated mainly by platelet antibodies. $T$ follicular helper (TFH) cells have demonstrated important roles in autoimmune diseases. The aim of this study is to explore the might role of TFH cells in the patients of ITP.

Methods: Twenty-three ITP patients and 12 healthy controls $(\mathrm{HC})$ were enrolled in this study. The frequency of circulating TFH cells in both the patients and $\mathrm{HC}$ was analyzed by flow cytometry. Serum interleukin (IL)-21 and IL-6 levels were measured using ELISA, and platelet antibodies were tested using a solid phase technique. Additionally, IL-21, IL-6, Bcl-6 and c-Maf mRNA expressions in peripheral blood mononuclear cells (PBMCs) were detected using real-time PCR.

Results: The percentages of circulating $\mathrm{CXCR}^{+} \mathrm{CD}^{+} \mathrm{TFH}$ cells with ICOS ${ }^{\text {high }}$ or PD- $1^{\text {high }}$ expression were significantly higher in the ITP patients than in the HC. Moreover, the frequencies of circulating CXCR5 ${ }^{+} \mathrm{CD} 4^{+} \mathrm{TFH}$ cells with inducible costimulator (ICOS) ${ }^{\text {high }}$ or programmed death-1 $(\text { PD- } 1)^{\text {high }}$ expression were notably higher in ITP with platelet-antibody-positive (ITP (+) ) patients than in ITP with platelet-antibody-negative ( ITP (-) ) patients and HC, as were the serum IL-2I and IL-6 levels (significant). Moreover, a positive correlation was found between the CXCR5 ${ }^{+}$CD $4^{+}$TFH cells with ICOS ${ }^{\text {high }}$ or PD- $1^{\text {high }}$ expression and the serum IL-21 levels of ITP (+) patients. Additionally, the mRNA expression levels of IL-21, IL-6, Bcl-6 and c-Maf were significantly increased in ITP patients, especially in ITP $(+)$ patients.

Conclusions: This study demonstrated TFH cells and effector molecules might play an important role in the pathogenesis of ITP, which are possible therapeutic targets in ITP patients.
\end{abstract}

Key words: platelet antibody; $\mathrm{T}$ follicular helper cell; idiopathic thrombocytopenic purpura; autoimmune disease

\section{Introduction}

Idiopathic thrombocytopenic purpura (ITP), characterized by a diminished peripheral platelet count $\left(<100 \times 10^{9} / \mathrm{L}\right)$ caused by platelet destruction with an increased risk of mucocutaneous bleeding, is a primary autoimmune disease [1]. Typical features of patients with ITP include skin petechiae and bleeding in the mucous membranes or internal organs that are easily manifested if the platelet count falls below $20 \times$ $10^{9} / \mathrm{L}$, although clinical symptoms are not obvious for most ITP patients. The diagnosis of ITP depends on 
clinical characteristics and the laboratory examinations conducted, as well as the ability to exclude other agents associated with thrombocytopenia $[2,3]$.

Platelet autoantibodies to specific membrane glycoproteins mediate platelet destruction and are a major agent in the pathogenetic mechanism of ITP that includes antibody-mediated cell-mediated platelet destruction and the suppression of megakaryopoiesis [4-6]. With ITP, the immunoglobulin (Ig) G autoantibodies derived from auto-reactive B cells can recognize and bind to one or more glycoproteins (GPs) on the surface of platelets, including GP IIb/IIIa, GP Ib/IX, and GP Ia/IIa; approximately 75\% are located on the platelet membrane glycoprotein GP $\mathrm{IIb} / \mathrm{III}$ a or GP Ib/IX complex [5]. These complexes can be easily swallowed and destroyed by the reticuloendothelial system by binding to $F_{C} \gamma$ receptors expressed on monocytes and macrophages, leading to a diminished peripheral platelet count [7]. However, little is known about how B cells produce specific auto-antibodies that are activated in patients with ITP and which $T$ cell type could induce B cells to produce antibodies in ITP patients.

$\mathrm{T}$ follicular helper (TFH) cells may play a critical role in regulating the humoral immune response that occurs with autoimmune diseases, infectious diseases, and tumors [8]. As a new subset, TFH cells regulate the immune process of antigen-specific B-cells and are characterized by the expression of molecules such as chemokine (C-X-C motif) receptor 5 (CXCR5), inducible costimulator (ICOS), programmed death-1 (PD-1), interleukin (IL)-21 receptor (IL-21R), IL-6 receptor (IL-6R), Bcl-6 and c-Maf [9-12].

TFH cells regulate B-cell responses that could produce appropriate specific antibodies [13]. High-level IL-21 excretion is a distinctive characteristic of TFH cells. Deficiency in IL-21 or IL-21R affects the evolution of B-cell-mediated immunity with an impaired isotype switch [14, 15]. PD-1 and ICOS, which are in the CD28 family, are two distinguishing molecules that have closely related functions in TFH cells [13, 16-20]. Bcl-6 and c-Maf are considered two important transcriptional factors of TFH cells that guide differentiation and controls the transcriptional signature of TFH cells $[12,21]$. In addition, some studies have defined ICOShigh CXCR5 ${ }^{+} \mathrm{CD}^{+}$and/or PD-1 ${ }^{\text {high }}{ }^{2 X C R} 5^{+} \mathrm{CD}^{+} \mathrm{T}$ cells as markers of circulating TFH cells that are closely correlated with the expression of antibodies in systemic lupus erythematosus (SLE) and Sjögren's syndrome [22].

Based on these findings, we hypothesized that circulating TFH cells may play an important role in regulating the production of platelet antibodies in patients with ITP. In addition, whether circulating TFH cells play a role in the pathogenesis of ITP re- mains unknown. Thus, we explored the role of circulating TFH cells in patients with ITP. We found that the percentage of circulating $\mathrm{CXCR} 5^{+} \mathrm{CD} 4{ }^{+} \mathrm{TFH}$ cells with ICOShigh or PD-1 high expression was significantly higher in ITP patients than in healthy controls (HC). Moreover, notably higher frequencies of circulating $\mathrm{CXCR}^{+} \mathrm{CD}^{+} \mathrm{TFH}$ cells with ICOShigh or PD-1 high expression, along with higher serum IL-21 and IL-6 levels (significant) were seen in ITP $(+)$ patients than in ITP (-) patients and HC. In addition, a positive correlation was found between $\mathrm{CXCR} 5^{+} \mathrm{CD} 4{ }^{+} \mathrm{TFH}$ cells with ICOShigh or PD-1 high expression and serum IL-21 cytokine levels in ITP $(+)$ patients. Although serum IL-21 and IL-6 levels were notably higher than that of $\mathrm{HC}$, the significant correlation was not found between serum IL-6 level and these parameters (data not shown). Furthermore, the mRNA expression levels of IL-21, IL-6, Bcl-6 and c-Maf were significantly higher in ITP $(+)$ patients than in ITP (-) patients and HC. Our results suggest that a higher frequency of circulating TFH cells in ITP patients might play a critical role in the pathogenesis of ITP.

\section{Methods}

\section{Patient demographics}

Using the criterion of the International Working Group of ITP defined in 2009 [23], we analyzed 23 patients diagnosed with chronic ITP in the Department of Hematology at our hospital. The clinical and laboratory characteristics of the 23 ITP patients and the $12 \mathrm{HC}$ subjects are presented in Table 1. All ITP patients had been diagnosed for about 24 months. None of the ITP patients had undergone a splenectomy. For about 2 months during this study, all the ITP patients did not have any other relevant diseases during the recent 3 months and were not prescribed any corticosteroids at the time they were analyzed. None of the HC (none of their family members) had a history of hematologic or autoimmune diseases, had ever received blood therapy, or had a history of blood transfusions. In contrast, all the ITP patients had local purpura of the skin and mucous membranes, had a history of platelet transfusions (at a minimum), and had a history of drug (dexamethasone, prednisolone, or prednisone) treatments. Patients with other hematologic diseases were excluded. All the samples were obtained according to the regulations and approval of the First Affiliated Hospital of Zhejiang University.

\section{Cell isolation}

Peripheral whole blood samples were obtained from the HC and ITP patients, and peripheral blood mononuclear cells (PBMC) were isolated using density gradient centrifugation with Ficoll-Hypaque solution (CL5020, CEDARLANE, The Netherlands). 
TABLE 1. Clinical characteristics of 23 recruited patients with ITP

\begin{tabular}{lllllll}
\hline Group & M/F & Age (years) & RBC $\left(\times 10^{12} / \mathrm{L}\right)$ & WBC $\left(\times 10^{9} / \mathrm{L}\right)$ & $\begin{array}{l}\text { PLT }\left(\times 10^{9} / \mathrm{L}\right) \\
\begin{array}{l}\text { Times of PLT } \\
\text { transfusions }\end{array}\end{array}$ & $\begin{array}{l}\text { ITP treatment history } \\
\text { of drugs }\end{array}$ \\
\hline HC & $6 / 6$ & $44 \pm 3.2$ & $5.2 \pm 0.6$ & $6.9 \pm 1.8$ & $176 \pm 30.6$ & 0 \\
ITP $(+)$ & $6 / 7$ & $46 \pm 11.6$ & $3.5 \pm 1.2$ & $2.6 \pm 1.6$ & $40 \pm 9.8$ & $6 \pm 0$ \\
ITP (-) & $5 / 5$ & $43 \pm 14.8$ & $3.3 \pm 1.4$ & $3.1 \pm 1.3$ & $44 \pm 12.2$ & $8 \pm 2.3$ \\
\hline
\end{tabular}

Note: data correspond to the arithmetic mean \pm SD. M/F: male/female; PLT: platelet; WBC: white blood cell; RBC: red blood cell; HC: healthy controls; ITP (+): ITP with platelet-antibody-positive; ITP (-): ITP with platelet-antibody-negative.

\section{Enzyme-linked immunosorbent assay analysis}

We separated the serum from the blood of all patients and then used those samples to test for the presence of cytokines IL-21 and IL-6 with an enzyme-linked immunosorbent assay (ELISA; BioLegend ELISA kit with pre-coated plate; BioLegend, San Diego, CA, USA). Each step was performed according to the manufacturer's protocol.

\section{Detection of platelet antibody}

For the ITP patients and HC, the platelet antibodies were tested according to the protocol of Platelet Crossmatch (MASPAT kit; K1360; Sanquin, Amsterdam, The Netherlands). A monolayer of donor platelets was immobilized by centrifugation onto the surfaces of microplate wells coated with platelet-specific mouse monoclonal antibody. Patient serum and low-ionic strength solution (LISS) were incubated in the appropriate wells, allowing serum antibodies to bind to the immobilized platelet monolayer. After incubation, non-bound serum components were removed by washing. Platelet-bound antibodies were detected with the addition of mouse monoclonal anti-human IgG and human IgG-sensitized erythrocytes. Positive reactions were characterized by the adherence of MASPAT indicator red cells throughout the well surfaces, whereas negative reactions produced discrete pellets of MASPAT indicator red cells in the middle of the wells.

\section{Flow cytometry analysis}

Human PBMCs were washed with phosphate-buffered saline (PBS) and immunostained with APC mouse anti-human CD4, FITC anti-human ICOS, and PerCP-Cy ${ }^{\mathrm{TM}}$ 5.5-mouse anti-human CD279 (PD-1) (BD Biosciences, San Diego, CA, USA), as well as PE anti-human CD185 (CXCR5) (BioLegend). Isotype-matched antibody controls were used in all procedures; these included FITC mouse IgG1, $\mathrm{K}$ isotype control Alexa, and mouse IgG1 kappa isotype control APC. All the staining procedures were performed according to the manufacturers' protocols, and the stained cells were analyzed using an FACS Calibur flow cytometer and CELLQUEST software (Becton Dickinson, Sparks, MD, USA). The remaining cells were saved in TRIzol reagent (Invitrogen, Carlsbad, CA, USA) for real-time polymerase chain reaction (PCR).

\section{RNA extraction and real-time PCR}

To detect the mRNA expression levels of IL-6, IL-21, and Bcl-6, the total RNA from the PBMCs was extracted using TRIzol reagent (Invitrogen). Next, cDNA was synthesized using a reverse transcription reagent kit (Toyobo, Osaka, Japan) according to the manufacturer's protocol. Real-time PCR was performed in triplicate using Takara SYBR Supermix (Takara, Dalian, China) and an ABI 7500 analysis system (Applied Biosystems, Foster City, CA, USA). The amplification conditions were as follows: $5 \mathrm{~min}$ at $95^{\circ} \mathrm{C}$ for denaturation, and then 40 cycles at $95^{\circ} \mathrm{C}$ for $10 \mathrm{~s}$ and $60^{\circ} \mathrm{C}$ for $40 \mathrm{~s}$. The fluorescence values were collected at $60^{\circ} \mathrm{C}$. The primer sequences were as follows [24, 25]:

IL-21: sense, 5'-CACAGACTAACATGCCCTT CAT-3', antisense, 5'-GAATCTTCACTTCCGTGTG TTCT-3';

IL-6: sense, 5'-AGACAGCCACTCACCTCTTC AG-3', antisense, 5'-TTCTGCCAGTGCCTCTTTG CTG-3';

Bcl-6: sense, 5'-CATGCAGAGATGTGCCTCCA CA-3', antisense, 5'-TCAGAGAAGCGGCAGTCA CACT-3';

c-Maf: sense, 5'-AGCGGCTTCCGAGAAAAC3', antisense, 5'-ACTTGCGAGTGGGCTCAG-3'.

Each gene was standardized using the expression of glyceraldehyde 3-phosphate dehydrogenase (GAPDH) with the following primers:

Sense, 5'-GTCTCCTCTGACTTCAACAGCG-3'; antisense, 5'-ACCACCCTGTTGCTGTAGCCAA-3'.

We analyzed the data using ABI 7500 software, v2.0.5 (Applied Biosystems).

\section{Statistical analysis}

All experiments were performed in triplicate except for the flow cytometry. One-way analysis of variance was used to determine whether an overall variation existed with statistical significance among the different groups. Student's unpaired or paired $t$ test was appropriately chosen. Correlations between variables were determined using Spearman's correla- 
tion coefficient. Data were analyzed using GraphPad Prism 5 software (GraphPad Software, Inc, San Diego, CA, USA).

\section{Results}

\section{Clinical data of patients with ITP}

ITP is an acquired immune-mediated disease characterized by a decrease in peripheral platelet count, and depending on the degree of thrombocytopenia, an increased risk of bleeding [26]. In the present study, 23 ITP patients were recruited and defined by clinical symptoms and platelet counts. We found that the peripheral white blood cells (WBCs), red blood cells (RBCs), and platelet counts of the ITP patients were significantly lower than those of the HC, and each ITP patient had local purpura of the skin and mucous membranes. Moreover, 13 of the 23 ITP patients were ITP $(+)$, and those patients had significantly more platelet transfusions than the ten ITP (-) patients $(p<0.0001)$, although there was not significantly different of platelet counts between ITP $(+)$ and ITP $(-)$ patients $(p=0.5543)$. Additionally, the ITP $(+)$ patients had more treatment experiences with drugs (such as dexamethasone and prednisone) than that of the ITP $(-)$ patients $(p=0.0118)$ (Table 1$)$.

\section{Increased frequency of circulating CXCR5+CD4+TFH cells in ITP patients}

Recent studies have shown that $\mathrm{CXCR}^{+} \mathrm{CD}^{+} \mathrm{TFH}$ cells play a critical role in regulating antibody response, and circulating CXCR5 ${ }^{+} \mathrm{CD} 4{ }^{+} \mathrm{TFH}$ cells with ICOShigh expression, PD-1 $1^{\text {high }}$ expression, or both are closely correlated with the expression of antibodies in many diseases $[20,24,27]$. Some studies have implied that platelet antibody-mediated platelet destruction plays an important role in the pathogenetic mechanisms of ITP [28]. However, little is known about the role of TFH cells in patients with ITP.

To explore the potential role of circulating TFH cells in patients with ITP, the frequency of circulating CXCR5 ${ }^{+} \mathrm{CD} 4{ }^{+} \mathrm{TFH}$ cells and percentages of ICOShigh $\mathrm{CXCR}^{+} \mathrm{CD}^{+}$and $\mathrm{PD}-1^{\text {high }} \mathrm{CXCR} 5^{+} \mathrm{CD} 4^{+} \mathrm{TFH}$ cells were analyzed using flow cytometry (Figure 1). We investigated using $\mathrm{CD}^{+} \mathrm{T}$ cells from human PBMC and analyzed CXCR5 ${ }^{+} \mathrm{T}$ cells to delimit TFH cells in peripheral blood from the ITP $(+)$ patients or ITP $(-)$ patients, and HC (Figure 1A-I). The percentages of circulating $\mathrm{CXCR}^{+} \mathrm{CD} 4^{+} \mathrm{TFH}$ cells, ICOShigh$\mathrm{CXCR}^{+}{ }^{+} \mathrm{CD} 4{ }^{+} \mathrm{TFH}$ cells, and $\mathrm{PD}-1^{\text {high }} \mathrm{CXCR} 5^{+} \mathrm{CD} 4^{+}$ TFH cells were significantly higher in ITP $(+)$ patients than those of ITP (-) patients and HC, respectively (Figure $1 \mathrm{~J}-\mathrm{L}$ ). However, the percentages of circulating CXCR5 ${ }^{+} \mathrm{CD}^{+} \mathrm{TFH}$ cells, ICOShigh ${ }^{+} \mathrm{CXCR}{ }^{+} \mathrm{CD} 4{ }^{+} \mathrm{TFH}$ cells, and PD-1 high CXCR5 ${ }^{+} \mathrm{CD} 4{ }^{+} \mathrm{TFH}$ cells were not significantly different between HC and ITP (-) patients (Figure $1 \mathrm{~J}-\mathrm{L}$ ). In addition, to further analyze whether the increased TFH cells had also high expression of ICOS or PD-1 molecules, Our study revealed that a strongly positive correlation was observed among CXCR5 ${ }^{+} \mathrm{CD} 4^{+} \mathrm{TFH}$ cells, ICOShigh$\mathrm{CXCR}^{+}{ }^{+} \mathrm{CD} 4{ }^{+} \mathrm{TFH}$ cells, and PD-1 high CXCR5 ${ }^{+}$ CD4 ${ }^{+}$TFH cells in ITP $\left(^{+}\right.$) patients (Figure $2 \mathrm{C}, \mathrm{F}, \mathrm{I}$ ), but no large difference was found in HC or ITP (-) patients (Figure 2 A, D, E, G, H). There was only a positive and modest correlation between CXCR5 ${ }^{+} \mathrm{CD} 4{ }^{+} \mathrm{TFH}$ cells and ICOShigh $\mathrm{CXCR} 5^{+} \mathrm{CD} 4+\mathrm{TFH}$ cells in ITP (-) patients (Figure 2B).

\section{Serum cytokines levels correlate with circu- lating TFH cells in ITP patients}

Recent studies have indicated that the cytokines IL-6 and IL-21 play important roles in the differentiation and function of TFH cells and in response to antibodies [29, 30].

The serum IL-6 and IL-21 levels were significantly higher in ITP patients than HC, irrespective of their status for platelet antibody, though the levels of these cytokines were highest in ITP $(+)$ patients (Figure $3 \mathrm{~A}, \mathrm{~B})$. Some studies have demonstrated that the levels of both IL-21 and IL-6 are significantly associated with the frequency of circulating TFH cells [29]. In the present study, the serum IL-21 levels were strongly and positively correlated with the percentages of $\mathrm{CXCR} 5^{+} \mathrm{CD} 4{ }^{+} \mathrm{TFH}$ cells, PD- $1^{\text {high }} \mathrm{CXCR} 5^{+} \mathrm{CD} 4^{+}$ TFH and ICOShigh CXCR5 ${ }^{+} \mathrm{CD} 4^{+} \mathrm{TFH}$ cells in ITP $\left(^{+}\right)$ patients, respectively (Figure $3 \mathrm{E}, \mathrm{H}, \mathrm{K}$ ). However, there were no correlation between IL-21 levels and these parameters in HC (Figure 3 C, F, I). Moreover, a moderate and positive correlation was found between the frequency of ICOShigh $C X C R 5^{+} \mathrm{CD} 4^{+} \mathrm{TFH}$ in ITP (-) patients (Figure $3 \mathrm{~J}$ ), although there was no significant correlation between serum IL-21 levels and $\mathrm{CXCR}^{+}{ }^{+} \mathrm{CD} 4^{+} \mathrm{TFH}$ cells or PD- $1^{\text {high }} \mathrm{CXCR} 5^{+} \mathrm{CD} 44^{+} \mathrm{TFH}$ (Figure 3 D, G). Unfortunately, we did not find any relation between serum IL-6 levels and these parameters in the HC and ITP (+) or ITP (-) patients, respectively (data not shown).

\section{Increased mRNA expression of IL-2 1, IL-6, Bcl-6 and c-Maf in ITP patients}

Cytokines IL-21 and IL-6, as well as the transcriptional factors of Bcl-6 and c-Maf, play crucial roles in the generation, differentiation and function of TFH cells $[12,29,31]$. We assessed the mRNA expression of Bcl-6, c-Maf, IL-21, and IL-6 mRNA in HC $(\mathrm{n}=12)$ and ITP $(-)$ patients $(\mathrm{n}=10)$ and ITP $(+)$ patients $(\mathrm{n}=13)$. The mRNA expression of IL-21 was higher in antibody-positive patients than in $\mathrm{HC}$ and 
ITP (-) patients, but no difference was found between the ITP (-) group and HC (Figure 4. A). The IL-6, Bcl-6 and c-Maf mRNA expressions were all notably higher in ITP $(+)$ patients and ITP (-) than that in HC, with a
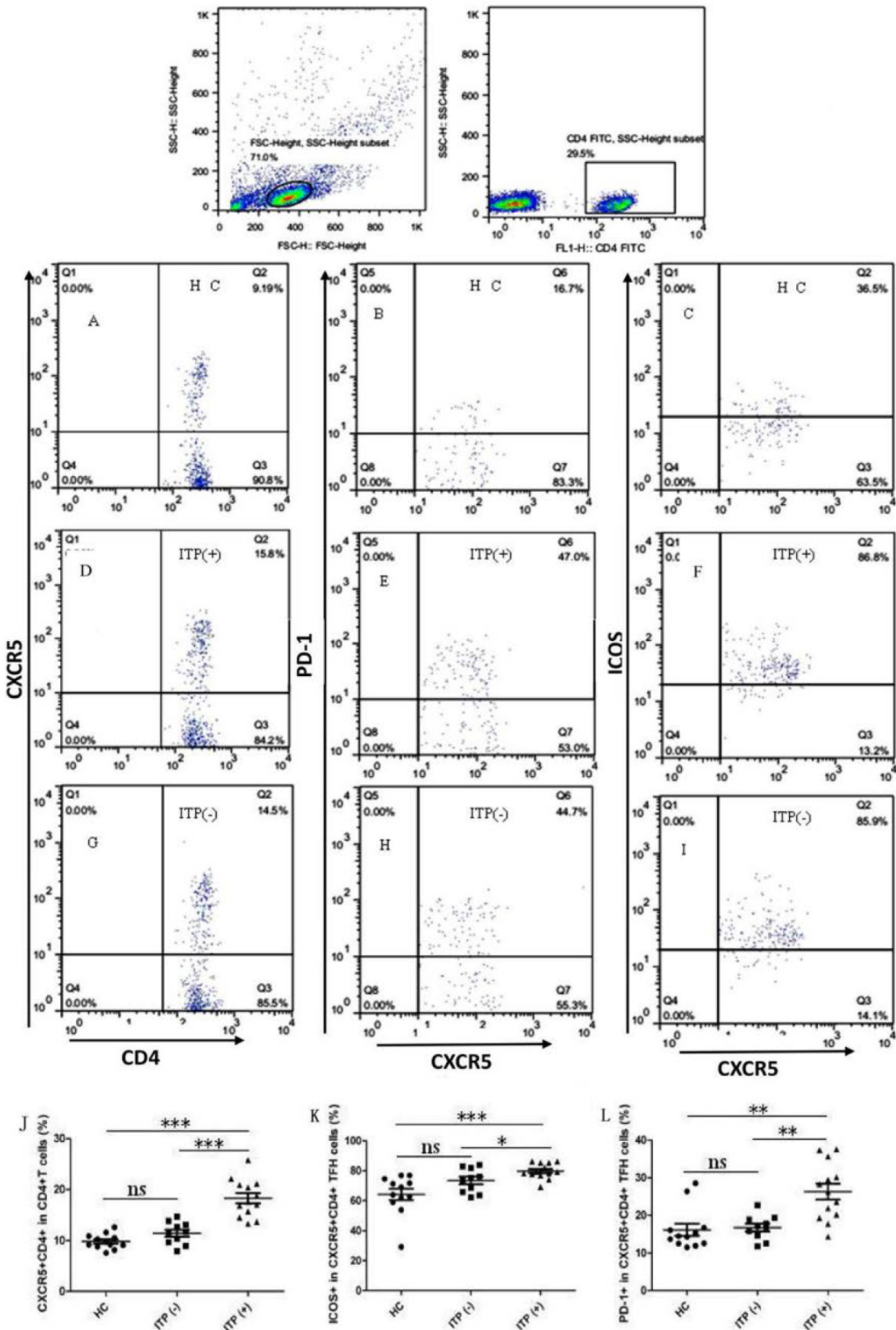

Fig. 1. Increased percentages of circulating $\mathrm{CXCR}^{+} \mathrm{CD} 4^{+} \mathrm{TFH}$ cells in the peripheral blood of ITP patients. Peripheral blood mononuclear cells (PBMC) from 23 patients with ITP and 12 health control $(\mathrm{HC})$ were isolated and stained with labeled antibodies, analyzed by flow cytometry as described in the Materials and Methods section. The cells were gated initially on lymphocytes (upper left) and then on CD4 ${ }^{+} T$ cells (upper right). (A, D, G):

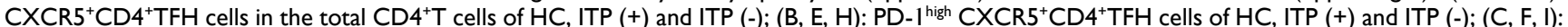
ICOS high CXCR5 $5^{+}$CD4 $4^{+}$TFH cells of HC, ITP (+) and ITP (-); (J-L): The analysis of percentages of CXCR5 $5^{+}$cells on CD4 $4^{+}$T cells, ICOS high and PD- $1^{\text {high }}$ cells on $\mathrm{CXCR5}^{+} \mathrm{CD} 4^{+} \mathrm{TFH}$ cells from HC, ITP $(+)$ and ITP $(-)$ patients. Each data point represents an individual subject. The horizontal lines show means. *, $p<0.05 ; * *, p<0.01 ; * * *, p<0.001 ;$ ns, no significant difference. 

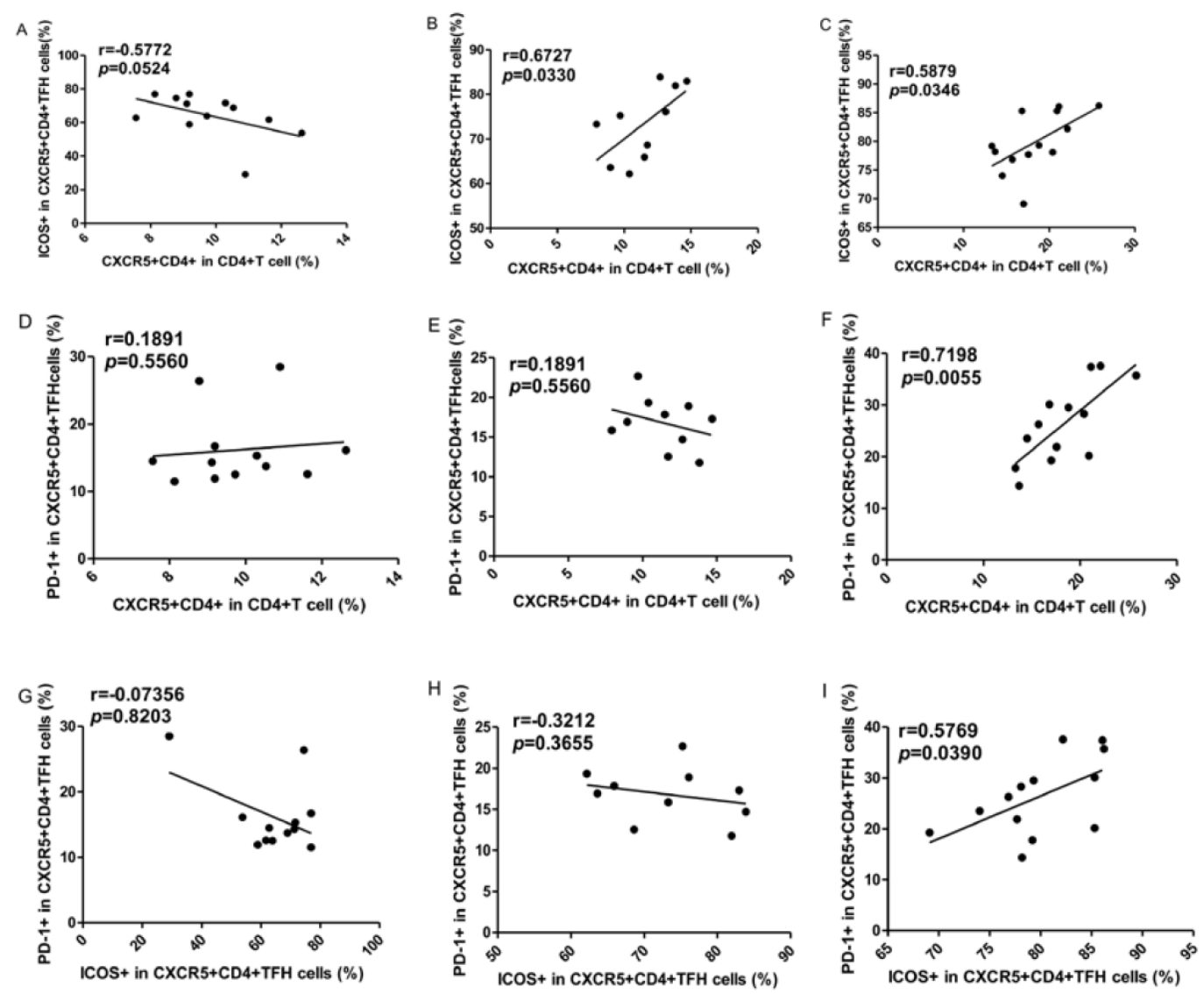

Fig. 2. Correlations of circulating TFH cells with $I C O S^{\text {high }}$ and PD-1 $1^{\text {high }}$ expression in ITP patients. (A, D, G): Relationship among the percentages of

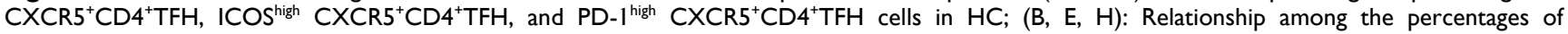
CXCR5 ${ }^{+} C D 4^{+} T F H$, ICOS high $C X C R 5^{+} C D 4^{+} T F H$, and PD- $1^{\text {high }}$ CXCR5 ${ }^{+} C D 4^{+} T F H$ cells in ITP (-); (C, F, I): Relationship among the percentages of

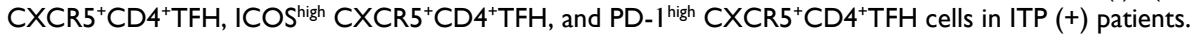

\section{Discussion}

Chronic ITP is an immune-mediated disorder characterized by a transient decrease in or persistently decreased platelet count caused by the platelet destruction, and the platelet antibody-mediated platelet destruction is a major causative factor for the pathogenetic mechanism of ITP $[1,32,33]$. In the current study, clinical features and laboratory evidences such as the degree of thrombocytopenia or petechiae on the skin, platelet count and platelet antibody expression were accordant with previous reports [26, 32, 33]. We found that ITP $(+)$ patients had significantly more platelet transfusions than the ITP (-) patients, which implied that exogenetic platelet transfusions might be helpful to generate platelet antibodies that accelerate the platelet destruction that decreased platelet count. Therefore, the development of the ITP patient, especially the ITP $(+)$ patients, would be attenuated by the reduced frequencies of platelet transfusions. Additionally, the higher frequencies of drug treatments might be important to reduce disease severity of ITP $(+)$ patients compared to ITP (-) patients.

Recent studies have implied that humoral im- mune response plays an important role in the pathogenesis of ITP that involves a complex interaction among antigen-presenting cells (APCs), T helper (Th) cells, regulatory $\mathrm{T}$ (Treg) cells and B cells [34, 35]. Furthermore, increasing evidence has shown that cytotoxic CD8+T cells contribute to platelet destruction [36]. It is well known that TFH cells play pivotal roles in autoimmune diseases and virus infection [13, 37-39]. However, the role of TFH cells remains unclear in the development and progression of ITP. The expression of CXCR5 is required for TFH cell migration to follicular areas of B cell in germinal center (GC) such as spleen, and for the formation of GC which is impaired in the absence of CXCR5 [9-11, 40]. Interestingly, up-regulation of CXCR5 is beneficial to enhance the probability of antigen-specific contact between TFH cells and B cells in draining lymphoid tissue [13]. Our results indicated that evaluated percentages of circulating CXCR5 ${ }^{+} \mathrm{CD} 44^{+} \mathrm{TFH}$ cells characterized with ICOShigh and PD-1 1 high in peripheral blood from both ITP patients and HC were accordant with a previous definition of circulating TFH cells [22], and with another previous report showing an increased frequency of TFH cell with ICOShigh and 
PD-1 high in peripheral blood and spleen of ITP patient patients [40].

that is correlated with the expansion of GC in ITP
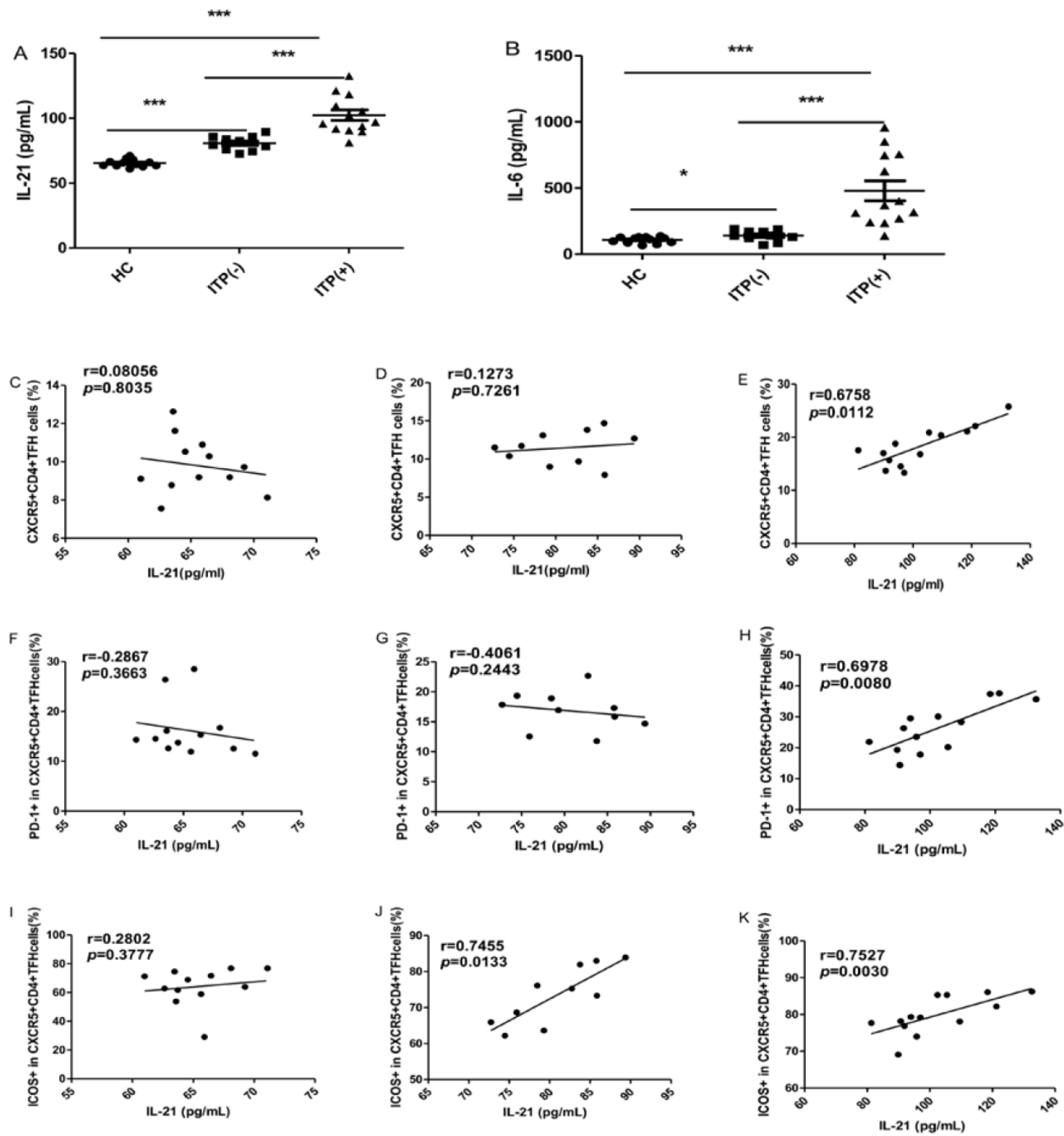

Fig. 3. Correlation of cytokine levels and circulating TFH cells in HC, and ITP (-) and ITP (+) patients. (A): Levels of serum IL-21 in HC, ITP (+) and ITP (-) patients; (B) Levels of serum IL-6 in HC, ITP $(-)$ and ITP $(+)$ patients; (C, D, E): Relationship of serum IL-21 levels and the percentage of CXCR5 ${ }^{+} C D 4^{+}$TFH cells in HC, ITP $(-)$ and ITP $(+)$ patients, respectively; $(F, G, H)$ : Relationship of serum IL-21 levels and the percentage of PD- $1^{\text {high }} C X C R 5^{+} C D 4^{+} T F H$ cells in HC, ITP (-) and ITP (+) patients, respectively; (I, J, K): Relationship of serum IL-21 levels and the percentage of ICOS ${ }^{\text {high }} \mathrm{CXCR5} 5^{+} \mathrm{CD} 4^{+} \mathrm{TFH}$ cells in HC, ITP (-) and ITP (+) patients, respectively. Additionally, there was no significant correlation of IL-6 levels and CXCR5 ${ }^{+}$CD4 $4^{+}$TFH cells in HC, ITP (-) and ITP $\left(^{+}\right)$patients, respectively, data was not shown. Data shown were the mean \pm SD. The horizontal lines show the median. $*, p<0.05 ; * *, p<0.01 ; * * *, p<0.001$. 

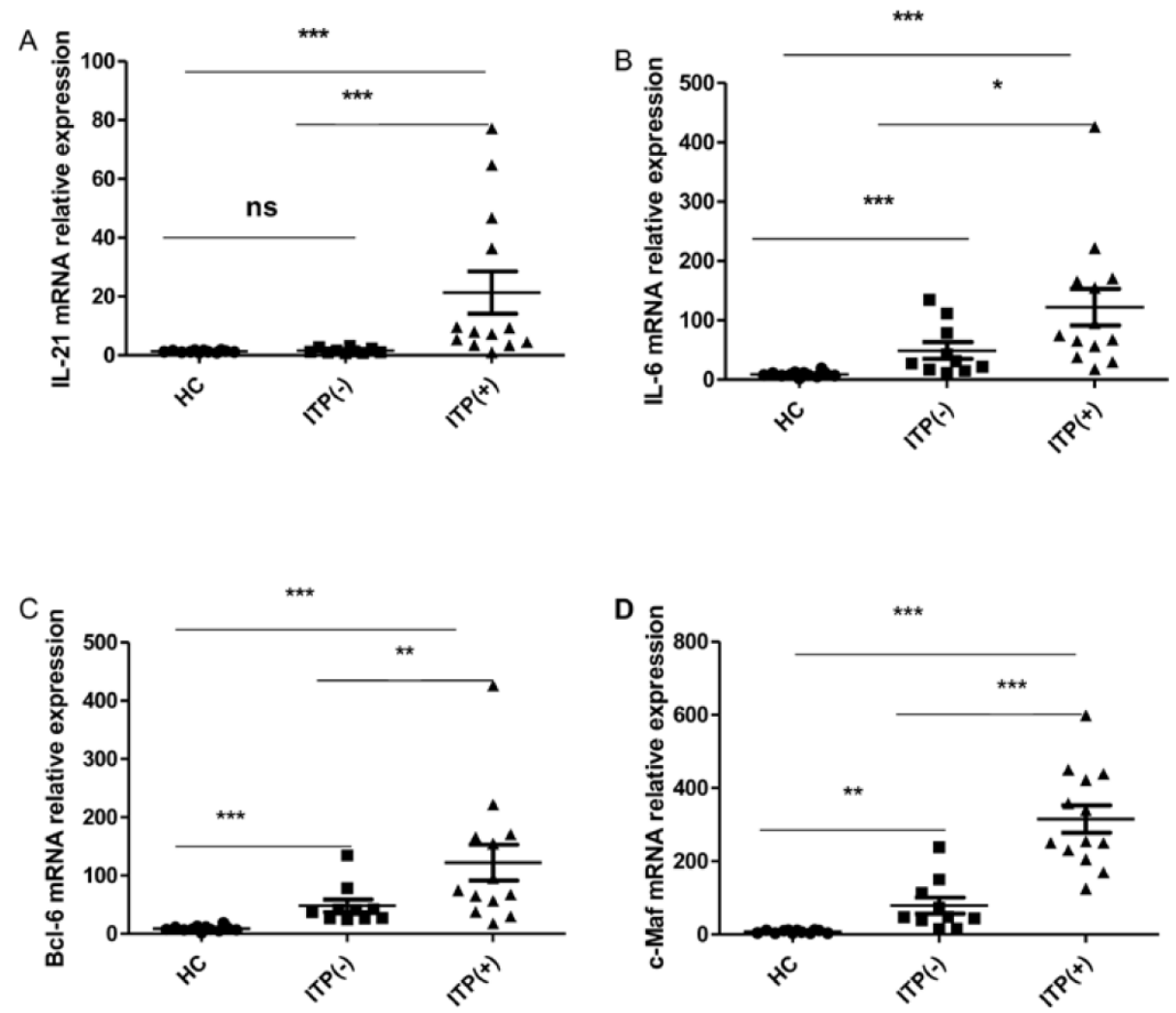

Fig. 4. Expression of IL-21, IL-6, Bcl-6 and c-Maf mRNA in PBMC from HC, ITP (-) and ITP (+) patients. (A): Levels of the relative expression of IL-2I mRNA in PBMC from HC, ITP (-) and ITP (+) patients; (B) Levels of the relative expression of IL-6 mRNA in PBMC from HC, ITP (-) and ITP (+) patients; (C) Levels of the relative expression of Bcl-6 mRNA in PBMC from HC, ITP (-) and ITP (+) patients; (D) Levels of the relative expression of c-Maf mRNA in PBMC from HC, ITP (-) and ITP (+) patients. Each data point represents an individual subject, and the data of each sample was an average value of three independent experiments with similar results. The horizontal lines show means. $*, p<0.05 ; * *, p<0.01 ; * * *, p<0.001$; ns, no significant difference.

ICOS and PD-1 molecules, belonging to the CD28 family members, are considered to be required for the function of TFH cell [16-20]. ICOS deficient human and mice are associated with severe decrease of CXCR5+CD4+TFH cells from GC and peripheral blood [17]. ICOS/ICOS-L deficient mice remarkably reduce antibody production and isotype switch, and severely disturbed GC formation [16-18]. Conversely, over-expression of ICOS in mice caused over-produced CXCR5+CD4+TFH cells, and induced exuberant GC reactions with breakthrough autoimmune disease, and notably promoted antibody production $[13,21]$. PD-1 molecule is considered to support negative regulation of chronically activated $\mathrm{T}$ cells, inhibiting excessive proliferation of TFH cells in GC $[8,19,20,27]$. However, PD-1 signaling can increase the number of TFH cell, decrease function of TFH by reducing its' associated cytokines such as IL-21, impacting GC formation and the quantity and quality of long-lived plasma cells in the absence of PD-1 [20]. Moreover, Recent studies have implicated that increased frequencies of circulating ICOShigh$\mathrm{CXCR}^{+} \mathrm{CD}^{+}$and/or PD- 1 high CXCR5 ${ }^{+} \mathrm{CD} 4{ }^{+} \mathrm{TFH}$ cells in many autoimmune diseases such as SLE, autoimmune thyroid disease (AITD), rheumatoid arthritis
(RA), and Sjögren's syndrome are closely associated with the production of auto-antibodies, TFH cells associated cytokines such as IL-21, which exacerbate the progression of those diseases [22, 24, 27, 29]. We also found that the frequencies of circulating $\mathrm{CXCR}^{+}{ }^{+} \mathrm{CD} 4^{+} \mathrm{TFH}$ cells with ICOShigh and PD-1high were highest in ITP $(+)$ patients,, although there was not differences of these parameters in between the ITP $(-)$ patients and $\mathrm{HC}$, respectively. Moreover, a strongly positive correlation was observed among the percentages of circulating $\mathrm{CXCR} 5{ }^{+} \mathrm{CD} 4{ }^{+} \mathrm{TFH}$ cells, ICOShigh ${ }^{2} \mathrm{CRR}^{+}{ }^{+} \mathrm{CD} 4{ }^{+} \mathrm{TFH}$ cells, and PD- $1^{\text {high }} \mathrm{CXCR}^{+} \mathrm{CD} 4^{+} \mathrm{TFH}$ cells in ITP $(+)$ patients. These results implied that higher expressions of ICOS and PD-1 on TFH cells in ITP patients, especially ITP $(+)$ patients, might be helpful to increase the quantity and quality of TFH cells that promote the induction of platelet auto-antibodies in the disorder ITP patients compared to HC, which were in agreement with previous reports $[22,24,40]$. In an addition, exogenetic platelet transfusions were a potential or partial explanation of increased TFH cells with higher expressions of ICOS and PD-1 in ITP $(+)$ patients. Moreover, drugs (such as dexamethasone, or prednisone) can effectively reduce the frequencies of TFH cells and 
attenuate disease severity in some autoimmune diseases [41, 42]. Higher frequencies of drug treatment in ITP $(+)$ patients were possibly responsible for inhibiting the increased TFH cells proportions in ITP $(+)$ patients compared to ITP (-) patients.

IL-21 is an important cytokine for germinal center formation, TFH cell proliferation and function, Ig-secreting B cells, and antibody response [37]. Moreover, IL-6 induces IL-21 secretion and TFH cell generation, and mice lacking both IL-6 and IL-21 cannot generate TFH cell-dependent immune responses [29]. Moreover, activated TFH cells with ICOS and/or PD-1 expression can produce abundant IL-21 cytokine by itself, and the levels of both IL-21 and IL- 6 are significantly associated with the frequency of circulating TFH cells with ICOS and/or PD-1 expression in some autoimmune diseases [31, 37, 43]. In the current study, we found significant differences about serum IL-21 and IL-6 levels among HC, ITP $(-)$ and ITP $(+)$ patients, which suggested that IL-21 and IL-6 might play important roles in ITP patients. Subsequent correlation analysis of between serum cytokine levels and the frequencies of TFH cells further indicated that increased IL-21 levels might play predominant role in the generation and differentiation of TFH cells with ICOShigh molecule compared to PD-1 molecule, and the productions of platelet antibodies in ITP patients, and IL-6 could play subsidiary roles in the pathogenesis of ITP patients, which are in accordance with previous report [38]. Additionally, these differences might be related to the number of ITP patients we evaluated, as well as other irregular antibodies, but not platelet antibodies. Bcl-6 and c-Maf transcription factor selectively expressed in TFH cells is essential for the generation and differentiation of TFH cells, and Bcl-6 expression is regulated by IL-6 and IL-21, c-Maf also induces CXCR5 expression and IL-21 secretion [12, 15, 29]. Additionally, Bcl-6 and c-Maf cooperate to induce the expressions of CXCR5, PD-1, and ICOS, and to instruct TFH cell differentiation [12]. PCR analyses implied that the increased mRNA expressions of IL-21, IL-6, and Bcl-6 and c-Maf in patients with ITP were possible explanations for the increased number of circulating $\mathrm{CXCR}^{+}{ }^{+} \mathrm{CD} 4{ }^{+} \mathrm{TFH}$ cells with ICOShigh and PD-1 high in these ITP patients, particularly in those ITP $(+)$ patients, which are consistent with prior studies $[12,24$, 40].

However, in this study, we did not observed any positive or negative correlation of between cytokine levels and percentages of TFH cell, or percentages of TFH cell and its associated molecules expressions in $\mathrm{HC}$, indicating the balance of internal environment depend on the balance of various cells and cytokines such as T cell, B cell and IL-2 1 in vivo contributes to the health of the body. Once the harmonious proportions of cells and cytokines are severely destroyed by internal or external factors such as pathogens and autoantigens, disease would occur such as ITP.

\section{Conclusions}

Our results had shown that the frequencies of circulating CXCR5 ${ }^{+} \mathrm{CD} 4^{+}$TFH cells with ICOShigh and PD-1 ${ }^{\text {high, }}$, expressions of TFH cell associated molecules such as IL-21 and Bcl-6 were significantly increased in ITP patients, particularly in ITP $(+)$ patients compared to $\mathrm{HC}$, which implied that endogenous platelet and/or more exogenetic platelet transfusions contributed to generation and differentiation of TFH cell that promoted the production of platelet antibody. High frequency of drug treatment (such as dexamethasone, or prednisone) might contribute to effectively reduce the frequencies of TFH cells and attenuate disease severity in ITP patients. Therefore, TFH cell might play a critical role in the pathogenesis of ITP, TFH cell and associated important molecules would be new therapeutic targets for ITP patients in the future. A large number of ITP patients, and the roles of TFH cells in regulating B-cell responses and antibody production in the pathogenesis of ITP will be investigated in the future study.

\section{Abbreviations}

ITP: Idiopathic thrombocytopenic purpura;

ITP (+): ITP with platelet-antibody-positive;

ITP (-): ITP with platelet-antibody-negative; GPs, glycoproteins; TFH, T follicular helper; IL, interleukin; IG, immunoglobulin; LISS, low-ionic strength solution; PBS, phosphate-buffered saline; CXCR5, chemokine receptor 5; ICOS, inducible costimulator; PD-1, programmed death-1; IL-21R, IL-21 receptor; IL-6R, IL-6 receptor; WBCs, white blood cells; RBCs, red blood cells; PCR, polymerase chain reaction.

\section{Acknowledgements}

This study was supported by the Natural Science Foundation of Zhejiang Province, China (LY13H080001), the Scientific Technology Projects of Health and Family Planning Commission of Zhejiang Province (2012KYA083), and the Scientific Research Projects of Education of Zhejiang Province, China (Y201224054).

\section{Authors' contributions}

Jue Xie, Dawei Cui, Jie Jin, and Hongyan Tong participated in the design of study. Yan Liu, Guoxiang Ruan, Lei Wang, Yun Lu and Huiming Yuan performed experimental work. Jue Xie, Dawei Cui, Jie Jin, and Yan Liu performed the statistical analysis and helped to draft the manuscript. Guoxiang Ruan, 
Hongyan Tong, and Yun Lu provided administrative support and funded experiments. All authors read and approved the final manuscript.

\section{Competing Interests}

The authors have declared that no competing interest exists.

\section{References}

1. Kuwana M. Helicobacter pylori-associated immune thrombocytopenia: clinical features and pathogenic mechanisms. World J Gastroenterol. 2014; 20: 714-23.

2. Metcalfe P. Platelet antigens and antibody detection. Vox Sang. 2004; 87 Suppl1: 82-6.

3. Sun L, Yu Z, Bu Y, Su J, Wang C, Cao L, et al. [The clinical studies of 51 patients with thrombotic thrombocytopenic purpura]. Zhonghua Xue Ye Xue Za Zhi. 2014; 35: 147-51.

4. [No authors listed]. Detection of platelet-reactive antibodies in patients who are refractory to platelet transfusions, and the selection of compatible donors. Vox Sang. 2003; 84: 73-88.

5. Cines DB, Blanchette VS. Immune thrombocytopenic purpura. N Engl J Med. 2002; 346: 995-1008.

6. Tripathi AK, Mishra S, Kumar A, Yadav D, Shukla A, Yadav Y. Megakaryocyte morphology and its impact in predicting response to steroid in immune thrombocytopenia. Platelets. 2014; 25: 526-31.

7. Stasi R. Pathophysiology and therapeutic options in primary immune thrombocytopenia. Blood Transfus. 2011; 9: 262-73.

8. Xiang D, Wang D, He Y, Xie J, Zhong Z, Li Z, et al. Caffeic acid phenethyl ester induces growth arrest and apoptosis of colon cancer cells via the beta-catenin/T-cell factor signaling. Anticancer Drugs. 2006; 17: 753-62.

9. Yang X, Yang J, Chu Y, Xue Y, Xuan D, Zheng S, et al. T follicular helper cells and regulatory $B$ cells dynamics in systemic lupus erythematosus. PloS one. 2014; 9: e88441.

10. Arnold CN, Campbell DJ, Lipp M, Butcher EC. The germinal center response is impaired in the absence of T cell-expressed CXCR5. Eur J Immunol. 2007; 37: $100-9$

11. Haynes NM, Allen CD, Lesley R, Ansel KM, Killeen N, Cyster JG. Role of CXCR5 and CCR7 in follicular Th cell positioning and appearance of a programmed cell death gene-1high germinal center-associated subpopulation. J Immunol. 2007; 179: 5099-108.

12. Kroenke MA, Eto D, Locci M, Cho M, Davidson T, Haddad EK, et al. Bcl6 and Maf cooperate to instruct human follicular helper CD4 T cell differentiation. J Immunol. 2012; 188: 3734-44

13. Fazilleau N, Mark L, McHeyzer-Williams LJ, McHeyzer-Williams MG. Follicular helper T cells: lineage and location. Immunity. 2009; 30: 324-35.

14. Ozaki K, Spolski R, Feng CG, Qi CF, Cheng J, Sher A, et al. A critical role for IL-21 in regulating immunoglobulin production. Science. 2002; 298: 1630-4.

15. Spolski R, Leonard WJ. IL-21 and T follicular helper cells. Int Immunol. 2010; 22. $7-12$

16. Hutloff A, Dittrich AM, Beier KC, Eljaschewitsch B, Kraft R, Anagnostopoulos $\mathrm{I}$, et al. ICOS is an inducible T-cell co-stimulator structurally and functionally related to CD28. Nature. 1999; 397: 263-6.

17. Bossaller L, Burger J, Draeger R, Grimbacher B, Knoth R, Plebani A, et al. ICOS deficiency is associated with a severe reduction of CXCR5+CD4 germinal center Th cells. J Immunol. 2006; 177: 4927-32.

18. Tafuri A, Shahinian A, Bladt F, Yoshinaga SK, Jordana M, Wakeham A, et al. ICOS is essential for effective T-helper-cell responses. Nature. 2001; 409: 105-9.

19. Ishida Y, Agata Y, Shibahara K, Honjo T. Induced expression of PD-1, a novel member of the immunoglobulin gene superfamily, upon programmed cell death. EMBO J. 1992; 11: 3887-95.

20. Good-Jacobson KL, Szumilas CG, Chen L, Sharpe AH, Tomayko MM, Shlomchik MJ. PD-1 regulates germinal center B cell survival and the formation and affinity of long-lived plasma cells. Nat Immunol. 2010; 11: 535-42.

21. Yu D, Rao S, Tsai LM, Lee SK, He Y, Sutcliffe EL, et al. The transcriptional repressor Bcl-6 directs T follicular helper cell lineage commitment. Immunity. 2009; 31: 457-68.

22. Simpson N, Gatenby PA, Wilson A, Malik S, Fulcher DA, Tangye SG, et al. Expansion of circulating $\mathrm{T}$ cells resembling follicular helper $\mathrm{T}$ cells is a fixed phenotype that identifies a subset of severe systemic lupus erythematosus. Arthritis Rheum. 2010; 62: 234-44.

23. Rodeghiero F, Stasi R, Gernsheimer T, Michel M, Provan D, Arnold DM, et al. Standardization of terminology, definitions and outcome criteria in immune thrombocytopenic purpura of adults and children: report from an international working group. Blood. 2009; 113: 2386-93.

24. Zhu C, Ma J, Liu Y, Tong J, Tian J, Chen J, et al. Increased frequency of follicular helper T cells in patients with autoimmune thyroid disease. J Clin Endocrinol Metab. 2012; 97: 943-50.

25. Li T, Xiao J, Wu Z, Qiu G. Over-expression of c-maf by chondrocytes in osteoarthritis. J Int Med Res. 2009; 37: 129-35.
26. Stasi R, Newland AC. ITP: a historical perspective. Br J Haematol. 2011; 153: 437-50.

27. Xu H, Wang X, Lackner AA, Veazey RS. PD-1(HIGH) Follicular CD4 T Helper Cell Subsets Residing in Lymph Node Germinal Centers Correlate with B Cell Maturation and IgG Production in Rhesus Macaques. Front Immunol. 2014; 5: 85 .

28. Cooper N, Stasi R, Cunningham-Rundles S, Cesarman E, McFarland JG, Bussel JB. Platelet-associated antibodies, cellular immunity and FCGR3a genotype influence the response to rituximab in immune thrombocytopenia. Br J Haematol. 2012; 158: 539-47.

29. Eto D, Lao C, DiToro D, Barnett B, Escobar TC, Kageyama R, et al. IL-21 and IL-6 are critical for different aspects of B cell immunity and redundantly induce optimal follicular helper CD4 T cell (Tfh) differentiation. PloS one. 2011; 6: e17739.

30. Park HJ, Kim DH, Lim SH, Kim WJ, Youn J, Choi YS, et al. Insights into the role of follicular helper T cells in autoimmunity. Immune Netw. 2014; 14: 21-9.

31. Nakayamada S, Poholek AC, Lu KT, Takahashi H, Kato M, Iwata S, et al. Type I IFN induces binding of STAT1 to Bcl6: divergent roles of STAT family transcription factors in the $\mathrm{T}$ follicular helper cell genetic program. J Immunol. 2014; 192: 2156-66.

32. Andersson PO, Wadenvik H. Chronic idiopathic thrombocytopenic purpura (ITP): molecular mechanisms and implications for therapy. Expert Rev Mol Med. 2004; 6: 1-17.

33. Najaoui A, Bakchoul T, Stoy J, Bein G, Rummel MJ, Santoso S, et al. Autoantibody-mediated complement activation on platelets is a common finding in patients with immune thrombocytopenic purpura (ITP). Eur J Haematol. 2012; 88: $167-74$.

34. Godeau B. B-cell depletion in immune thrombocytopenia. Semin Hematol. 2013; 50 Suppl 1: S75-82.

35. Nishimoto $T$, Satoh $T$, Takeuchi $T$, Ikeda $Y$, Kuwana M. Critical role of CD4(+)CD25(+) regulatory $\mathrm{T}$ cells in preventing murine autoantibody-mediated thrombocytopenia. Exp Hematol. 2012; 40: 279-89.

36. Nishimoto T, Kuwana M. CD4+CD25+Foxp3+ regulatory T cells in the pathophysiology of immune thrombocytopenia. Semin Hematol. 2013; 50 Suppl 1: S43-9.

37. Terrier B, Costedoat-Chalumeau N, Garrido M, Geri G, Rosenzwajg M, Musset $\mathrm{L}$, et al. Interleukin 21 correlates with $\mathrm{T}$ cell and $\mathrm{B}$ cell subset alterations in systemic lupus erythematosus. J Rheumatol. 2012; 39: 1819-28.

38. Wang J, Shan Y, Jiang Z, Feng J, Li C, Ma L, et al. High frequencies of activated $\mathrm{B}$ cells and $\mathrm{T}$ follicular helper cells are correlated with disease activity in patients with new-onset rheumatoid arthritis. Clin Exp Immunol. 2013; 174: $212-20$

39. Wu J, Cui D, Yang X, Lou J, Lin J, Ye X, et al. Increased frequency of circulating follicular helper T cells in children with hand, foot, and mouth disease caused by enterovirus 71 infection. J Immunol Res. 2014; 2014: 651872.

40. Audia S, Rossato M, Santegoets K, Spijkers S, Wichers C, Bekker C, et al. Splenic TFH expansion participates in B-cell differentiation and antiplatelet-antibody production during immune thrombocytopenia. Blood. 2014; 124: 2858-66.

41. Zhang L, Wang Y, Shi X, Zou H, Jiang Y. A higher frequency of CD4(+)CXCR5(+) T follicular helper cells in patients with newly diagnosed IgA nephropathy. Immunology letters. 2014; 158: 101-8.

42. Feng X, Wang D, Chen J, Lu L, Hua B, Li X, et al. Inhibition of aberrant circulating Tfh cell proportions by corticosteroids in patients with systemic lupus erythematosus. PloS one. 2012; 7: e51982.

43. Xie A, Buras ED, Xia J, Chen W. The Emerging Role of Interleukin-21 in Transplantation. J Clin Cell Immunol. 2012; Suppl 9: 1-7. 